\title{
Synthesis of $\left[\left(\eta^{5}-\mathrm{C}_{5} \mathrm{H}_{5}\right) \mathrm{Fe}(\mathrm{CO})_{2} \mathrm{BCl}_{2} \cdot \mathrm{NC}_{5} \mathrm{H}_{4}-4-\mathrm{Me}\right]$ from $\left[\left(\eta^{5}-\mathrm{C}_{5} \mathrm{H}_{5}\right) \mathrm{Fe}(\mathrm{CO})_{2} \mathrm{BCl}_{2}\right]$ : the first preparation of a Lewis acid-base adduct from a boryl complex
}

\author{
Holger Braunschweig, * Krzysztof Radacki, Fabian Seeler and George R. Whittell
}

1. Synthetic procedures and spectroscopic data

\section{General Considerations}

All manipulations were conducted either under an atmosphere of dry argon or in vacuo using standard

Schlenk line or glove-box techniques. Solvents (toluene and hexane) were purified by distillation from appropriate drying agents (sodium and sodium wire, respectively) under dry argon, immediately prior to use. IR spectra were recorded as toluene solutions between $\mathrm{KBr}$ plates on a Bruker Vector 22 FT-IRspectrometer. ${ }^{1} \mathrm{H}$ and ${ }^{13} \mathrm{C}$ NMR spectra were acquired on a Bruker AMX 400 NMR spectrometer at 400.14 MHz and 100.63 MHz, respectively, and referenced to external TMS via the residual protio solvent $\left({ }^{1} \mathrm{H}\right)$ or the solvent itself $\left({ }^{13} \mathrm{C}\right) .{ }^{11} \mathrm{~B}$ NMR spectra were recorded on a Bruker Avance 200 NMR spectrometer at $64.22 \mathrm{MHz}$ and referenced to external $\mathrm{BF}_{3} . \mathrm{OEt}_{2}$. Microanalyses for $\mathrm{C}, \mathrm{H}$ and $\mathrm{N}$ were performed on a Carlo Erba Elemental Analyzer 1106.

Synthesis of $\left[\left({ }^{5}-\mathrm{C}_{5} \mathrm{H}_{5}\right) \mathrm{Fe}(\mathrm{CO})_{2} \mathrm{BCl}_{2}\right](\mathbf{1})$. A Schlenk tube containing a suspension of $\mathrm{Na}\left[\left({ }^{5}-\right.\right.$ $\left.\left.\mathrm{C}_{5} \mathrm{H}_{5}\right) \mathrm{Fe}(\mathrm{CO})_{2}\right](1.43 \mathrm{~g}, 7.15 \mathrm{mmol})$ in toluene $(20 \mathrm{~mL})$ was cooled to $-78{ }^{\circ} \mathrm{C}$, evacuated and gaseous $\mathrm{BCl}_{3}(2.88 \mathrm{~g}, 24.6 \mathrm{mmol})$ introduced. The reaction mixture was slowly allowed to warm to $-30{ }^{\circ} \mathrm{C}$, at which point the orange coloured suspension began to darken. Stirring was continued at this temperature for $c a$. 1 hour, resulting in the formation of a cloudy, dark red solution. Subsequent removal of all volatiles in vacuo, at room temperature, afforded a dark red oil. Hexane $(20 \mathrm{~mL})$ was added and residual solids separated by centrifugation. The clear, red supernatant liquors were then decanted, reduced in volume by $c a .50 \%$ and cooled to $-60{ }^{\circ} \mathrm{C}$. After a period of $c a .16$ hours, the red coloured 
crystals of pure 1 that had formed were isolated and dried in vacuo (yield $0.58 \mathrm{~g}, 31 \%$ ). IR: 2026, 1974 $\mathrm{cm}^{-1}$, (CO). NMR $\left(\mathrm{C}_{6} \mathrm{D}_{6}\right):{ }^{1} \mathrm{H} 3.97\left(\mathrm{~s}, \mathrm{C}_{5} \mathrm{H}_{5}\right) ;{ }^{13} \mathrm{C}\left\{{ }^{1} \mathrm{H}\right\} 86.61\left(\mathrm{~s}, \mathrm{C}_{5} \mathrm{H}_{5}\right), 213.1$ (s, $\left.\mathrm{CO}\right) ;{ }^{11} \mathrm{~B}\left\{{ }^{1} \mathrm{H}\right\} 90.0$ (br s). $\mathrm{C}_{7} \mathrm{H}_{5} \mathrm{O}_{2} \mathrm{BFeCl}_{2}$ requires: C 32.50, $\mathrm{H} 1.95 \%$; found 32.59, $2.07 \%$.

Synthesis of $\left[\left({ }^{5}-\mathrm{C}_{5} \mathrm{H}_{5}\right) \mathrm{Fe}(\mathrm{CO})_{2} \mathrm{BCl}_{2} \cdot \mathrm{NC}_{5} \mathrm{H}_{4}-4-\mathrm{Me}\right](2)$. A solution of $\mathbf{1}(0.49 \mathrm{~g}, 1.89 \mathrm{mmol})$ in toluene (15 $\mathrm{mL}$ ) was cooled to $0{ }^{\circ} \mathrm{C}$ and a solution of 4-methylpyridine $(0.18 \mathrm{~g}, 1.89 \mathrm{mmol})$ in further toluene (5 $\mathrm{mL}$ ) added dropwise. The reaction mixture was allowed to warm, with stirring, to room temperature, and all volatiles were removed in vacuo to afford pure 2 in quantitative yield. IR: $1976,1916 \mathrm{~cm}^{-1}$, (CO). NMR $\left(\mathrm{C}_{6} \mathrm{D}_{6}\right):{ }^{1} \mathrm{H} 1.64\left(\mathrm{~s}, 3 \mathrm{H}, \mathrm{NC}_{5} \mathrm{H}_{4}-4-M e\right), 4.94\left(\mathrm{~s}, 5 \mathrm{H}, \mathrm{C}_{5} \mathrm{H}_{5}\right), 6.49\left(\mathrm{~d}, 2 \mathrm{H},{ }^{3} \mathrm{~J}_{\mathrm{CH}}=6.4 \mathrm{~Hz}, \mathrm{NC}_{5} \mathrm{H}_{2}-\right.$ 3,5- $\left.\mathrm{H}_{2}-4-\mathrm{Me}\right), 9.46\left(\mathrm{~d}, 2 \mathrm{H},{ }^{3} \mathrm{~J}_{\mathrm{CH}}=6.4 \mathrm{~Hz}, \mathrm{NC}_{3} \mathrm{H}_{4}-2,6-\mathrm{H}_{2}-4-\mathrm{Me}\right) ;{ }^{13} \mathrm{C}\left\{{ }^{1} \mathrm{H}\right\} 19.05\left(\mathrm{~s}, \mathrm{NC}_{5} \mathrm{H}_{4}-4-M e\right), 80.32(\mathrm{~s}$, $\left.\mathrm{C}_{5} \mathrm{H}_{5}\right), 123.7\left(\mathrm{~s}, \mathrm{NC}_{4}-4-C-\mathrm{H}_{4}-4-\mathrm{Me}\right), 142.4\left(\mathrm{~s}, \mathrm{NC}_{3}-3,5-C_{2}-\mathrm{H}_{4}-4-\mathrm{Me}\right), 151.8\left(\mathrm{~s}, \mathrm{NC}_{3}-2,6-C_{2}-\mathrm{H}_{4}-4-\mathrm{Me}\right)$; ${ }^{11} \mathrm{~B}\left\{{ }^{1} \mathrm{H}\right\} 18.8$ (s). $\mathrm{C}_{13} \mathrm{H}_{12} \mathrm{O}_{2} \mathrm{NBFeCl}_{2}$ requires: $\mathrm{C} 44.38 ; \mathrm{H} 3.44 ; \mathrm{N} 3.98 \%$. Found $\mathrm{C} 43.48 ; \mathrm{H} 3.42 ; \mathrm{N} 4.01$ $\%$. 


\section{Structural determinations}

Table 1. Crystal data and structure refinement for $\left[\left({ }^{5}-\mathrm{C}_{5} \mathrm{H}_{5}\right) \mathrm{Fe}(\mathrm{CO})_{2} \mathrm{BCl}_{2}\right](\mathbf{1})$.

Identification code

Empirical formula

Formula weight

Temperature

Wavelength

Crystal system

Space group

Unit cell dimensions

Volume

$\mathrm{Z}$

Density (calculated)

Absorption coefficient

$\mathrm{F}(000)$

Crystal size

Theta range for data collection

Index ranges

Reflections collected

Independent reflections

Completeness to theta $=26.15^{\circ}$

Absorption correction

Max. and min. transmission

Refinement method

Data / restraints / parameters

Goodness-of-fit on $\mathrm{F}^{2}$

Final $\mathrm{R}$ indices [I $>2 \operatorname{sigma}(\mathrm{I})]$

$\mathrm{R}$ indices (all data)

Largest diff. peak and hole kr012

C7 $\mathrm{H} 5 \mathrm{~B} \mathrm{Cl} 2 \mathrm{Fe} \mathrm{O} 2$

258.67

173(2) K

$71.073 \mathrm{pm}$

Triclinic

P'̄

$$
\begin{array}{ll}
\mathrm{a}=7.6434(17) \AA & \alpha=89.656(4)^{\circ} . \\
\mathrm{b}=8.4881(19) \AA & \beta=70.731(4)^{\circ} . \\
\mathrm{c}=8.843(2) \AA & \gamma=63.761(4)^{\circ} .
\end{array}
$$

4791.2(19) $\AA^{3}$

2

$1.793 \mathrm{Mg} / \mathrm{m}^{3}$

$2.088 \mathrm{~mm}^{-1}$

256

$0.05 \times 0.09 \times 0.11 \mathrm{~mm}^{3}$

2.47 to $26.15^{\circ}$.

$-9<=\mathrm{h}<=9,-10<=\mathrm{k}<=10,-10<=\mathrm{l}<=10$

10279

$1914\left[\mathrm{R}_{\mathrm{int}}=0.0312\right]$

$100.0 \%$

Empirical

1.000 and 0.873

Full-matrix least-squares on $\mathrm{F}^{2}$

1914 / 0 / 118

1.077

$\mathrm{R}_{1}=0.0300, \mathrm{wR}_{2}=0.0745$

$\mathrm{R}_{1}=0.0330, \mathrm{wR}_{2}=0.0764$

0.781 and -0.294 e. $\AA^{-3}$

Table 2. Atomic coordinates [ $\left.\mathrm{x} 10^{4}\right]$ and equivalent isotropic displacement parameters $\left[\AA^{2} \times 10^{3}\right]$ for $\mathbf{1} . \mathrm{U}_{\mathrm{eq}}$ is defined as one third of the trace of the orthogonalized $\mathrm{U}_{\mathrm{ij}}$ tensor.

\begin{tabular}{lcrrr}
\hline & $\mathrm{x}$ & $\mathrm{y}$ & $\mathrm{z}$ & $\mathrm{U}_{\text {eq }}$ \\
\hline $\mathrm{Fe}$ & $4918(1)$ & $3073(1)$ & $2023(1)$ & $25(1)$ \\
$\mathrm{B}$ & $7094(4)$ & $971(4)$ & $2384(3)$ & $32(1)$ \\
$\mathrm{Cl}(1)$ & $8199(2)$ & $-1235(1)$ & $1344(1)$ & $66(1)$ \\
$\mathrm{O}(1)$ & $7620(3)$ & $4723(3)$ & $1575(2)$ & $43(1)$ \\
$\mathrm{C}(1)$ & $6553(4)$ & $4078(3)$ & $1740(3)$ & $30(1)$ \\
$\mathrm{O}(2)$ & $6459(3)$ & $1584(3)$ & $-1375(2)$ & $46(1)$ \\
$\mathrm{C}(2)$ & $5870(3)$ & $2164(3)$ & $-41(3)$ & $31(1)$ \\
$\mathrm{Cl}(2)$ & $8166(1)$ & $992(1)$ & $3890(1)$ & $52(1)$ \\
$\mathrm{C}(11)$ & $2982(4)$ & $2620(4)$ & $4052(4)$ & $55(1)$ \\
$\mathrm{C}(12)$ & $2881(4)$ & $4279(5)$ & $4390(3)$ & $52(1)$ \\
$\mathrm{C}(13)$ & $2084(4)$ & $5351(4)$ & $3336(4)$ & $47(1)$
\end{tabular}




\begin{tabular}{lllll}
$\mathrm{C}(14)$ & $1704(4)$ & $4404(4)$ & $2361(3)$ & $43(1)$ \\
$\mathrm{C}(15)$ & $2266(4)$ & $2692(4)$ & $2761(4)$ & $47(1)$ \\
\hline
\end{tabular}

Table 3. Bond lengths $[\AA]$ and angles $\left[^{\circ}\right]$ for $\mathbf{1}$.

\begin{tabular}{|c|c|}
\hline $\mathrm{Fe}-\mathrm{C}(1)$ & $1.757(2)$ \\
\hline $\mathrm{Fe}-\mathrm{C}(2)$ & $1.761(2)$ \\
\hline Fe-B & $1.942(3)$ \\
\hline $\mathrm{Fe}-\mathrm{C}(11)$ & $2.074(3)$ \\
\hline $\mathrm{Fe}-\mathrm{C}(12)$ & $2.078(3)$ \\
\hline $\mathrm{Fe}-\mathrm{C}(15)$ & $2.083(3)$ \\
\hline $\mathrm{Fe}-\mathrm{C}(13)$ & $2.108(3)$ \\
\hline $\mathrm{Fe}-\mathrm{C}(14)$ & $2.113(2)$ \\
\hline $\mathrm{B}-\mathrm{Cl}(1)$ & $1.781(3)$ \\
\hline $\mathrm{B}-\mathrm{Cl}(2)$ & $1.783(3)$ \\
\hline $\mathrm{O}(1)-\mathrm{C}(1)$ & $1.141(3)$ \\
\hline $\mathrm{O}(2)-\mathrm{C}(2)$ & $1.139(3)$ \\
\hline $\mathrm{C}(11)-\mathrm{C}(12)$ & $1.405(5)$ \\
\hline $\mathrm{C}(11)-\mathrm{C}(15)$ & $1.410(5)$ \\
\hline $\mathrm{C}(12)-\mathrm{C}(13)$ & $1.393(4)$ \\
\hline $\mathrm{C}(13)-\mathrm{C}(14)$ & $1.361(4)$ \\
\hline $\mathrm{C}(14)-\mathrm{C}(15)$ & $1.401(4)$ \\
\hline $\mathrm{C}(1)-\mathrm{Fe}-\mathrm{C}(2)$ & $94.91(11)$ \\
\hline $\mathrm{C}(1)-\mathrm{Fe}-\mathrm{B}$ & $88.02(11)$ \\
\hline $\mathrm{C}(2)-\mathrm{Fe}-\mathrm{B}$ & $90.72(11)$ \\
\hline $\mathrm{C}(1)-\mathrm{Fe}-\mathrm{C}(11)$ & $133.86(13)$ \\
\hline $\mathrm{C}(2)-\mathrm{Fe}-\mathrm{C}(11)$ & $130.21(13)$ \\
\hline $\mathrm{B}-\mathrm{Fe}-\mathrm{C}(11)$ & $83.00(12)$ \\
\hline $\mathrm{C}(1)-\mathrm{Fe}-\mathrm{C}(12)$ & $99.70(11)$ \\
\hline $\mathrm{C}(2)-\mathrm{Fe}-\mathrm{C}(12)$ & $161.21(11)$ \\
\hline $\mathrm{B}-\mathrm{Fe}-\mathrm{C}(12)$ & $101.48(13)$ \\
\hline $\mathrm{C}(11)-\mathrm{Fe}-\mathrm{C}(12)$ & $39.55(13)$ \\
\hline $\mathrm{C}(1)-\mathrm{Fe}-\mathrm{C}(15)$ & $161.66(11)$ \\
\hline $\mathrm{C}(2)-\mathrm{Fe}-\mathrm{C}(15)$ & $97.08(11)$ \\
\hline $\mathrm{B}-\mathrm{Fe}-\mathrm{C}(15)$ & $105.61(12)$ \\
\hline $\mathrm{C}(11)-\mathrm{Fe}-\mathrm{C}(15)$ & $39.67(13)$ \\
\hline $\mathrm{C}(12)-\mathrm{Fe}-\mathrm{C}(15)$ & $66.03(11)$ \\
\hline $\mathrm{C}(1)-\mathrm{Fe}-\mathrm{C}(13)$ & $96.76(11)$ \\
\hline $\mathrm{C}(2)-\mathrm{Fe}-\mathrm{C}(13)$ & $127.72(12)$ \\
\hline $\mathrm{B}-\mathrm{Fe}-\mathrm{C}(13)$ & $140.34(13)$ \\
\hline $\mathrm{C}(11)-\mathrm{Fe}-\mathrm{C}(13)$ & $65.43(12)$ \\
\hline $\mathrm{C}(12)-\mathrm{Fe}-\mathrm{C}(13)$ & $38.88(13)$ \\
\hline $\mathrm{C}(15)-\mathrm{Fe}-\mathrm{C}(13)$ & 64.91(11) \\
\hline $\mathrm{C}(1)-\mathrm{Fe}-\mathrm{C}(14)$ & $125.54(11)$ \\
\hline $\mathrm{C}(2)-\mathrm{Fe}-\mathrm{C}(14)$ & $97.21(11)$ \\
\hline $\mathrm{B}-\mathrm{Fe}-\mathrm{C}(14)$ & $144.32(11)$ \\
\hline
\end{tabular}




\begin{tabular}{lc}
$\mathrm{C}(11)-\mathrm{Fe}-\mathrm{C}(14)$ & $65.37(11)$ \\
$\mathrm{C}(12)-\mathrm{Fe}-\mathrm{C}(14)$ & $64.62(11)$ \\
$\mathrm{C}(15)-\mathrm{Fe}-\mathrm{C}(14)$ & $38.99(12)$ \\
$\mathrm{C}(13)-\mathrm{Fe}-\mathrm{C}(14)$ & $37.64(11)$ \\
$\mathrm{Cl}(1)-\mathrm{B}-\mathrm{Cl}(2)$ & $110.83(15)$ \\
$\mathrm{Cl}(1)-\mathrm{B}-\mathrm{Fe}$ & $125.32(15)$ \\
$\mathrm{Cl}(2)-\mathrm{B}-\mathrm{Fe}$ & $123.79(15)$ \\
$\mathrm{O}(1)-\mathrm{C}(1)-\mathrm{Fe}$ & $179.1(2)$ \\
$\mathrm{O}(2)-\mathrm{C}(2)-\mathrm{Fe}$ & $179.1(2)$ \\
$\mathrm{C}(12)-\mathrm{C}(11)-\mathrm{C}(15)$ & $107.3(3)$ \\
$\mathrm{C}(12)-\mathrm{C}(11)-\mathrm{Fe}$ & $70.40(16)$ \\
$\mathrm{C}(15)-\mathrm{C}(11)-\mathrm{Fe}$ & $70.54(16)$ \\
$\mathrm{C}(13)-\mathrm{C}(12)-\mathrm{C}(11)$ & $107.7(3)$ \\
$\mathrm{C}(13)-\mathrm{C}(12)-\mathrm{Fe}$ & $71.71(15)$ \\
$\mathrm{C}(11)-\mathrm{C}(12)-\mathrm{Fe}$ & $70.05(16)$ \\
$\mathrm{C}(14)-\mathrm{C}(13)-\mathrm{C}(12)$ & $108.8(3)$ \\
$\mathrm{C}(14)-\mathrm{C}(13)-\mathrm{Fe}$ & $71.37(15)$ \\
$\mathrm{C}(12)-\mathrm{C}(13)-\mathrm{Fe}$ & $69.41(16)$ \\
$\mathrm{C}(13)-\mathrm{C}(14)-\mathrm{C}(15)$ & $109.0(3)$ \\
$\mathrm{C}(13)-\mathrm{C}(14)-\mathrm{Fe}$ & $70.99(15)$ \\
$\mathrm{C}(15)-\mathrm{C}(14)-\mathrm{Fe}$ & $69.39(14)$ \\
$\mathrm{C}(14)-\mathrm{C}(15)-\mathrm{C}(11)$ & $107.1(3)$ \\
$\mathrm{C}(14)-\mathrm{C}(15)-\mathrm{Fe}$ & $71.63(15)$ \\
$\mathrm{C}(11)-\mathrm{C}(15)-\mathrm{Fe}$ & $69.79(15)$ \\
\hline
\end{tabular}

Table 4. Anisotropic displacement parameters $\left[\AA^{2} \times 10^{3}\right]$ for $\mathbf{1}$. The anisotropic displacement factor exponent takes the form: $-2 \pi^{2}\left[h^{2} a^{* 2} U_{11}+\ldots+2 h k a * b * U_{12}\right]$

\begin{tabular}{llllrrr}
\hline & $\mathrm{U}_{11}$ & $\mathrm{U}_{22}$ & $\mathrm{U}_{33}$ & $\mathrm{U}_{23}$ & $\mathrm{U}_{13}$ & $\mathrm{U}_{12}$ \\
\hline & & & & & & \\
$\mathrm{Fe}$ & $23(1)$ & $23(1)$ & $27(1)$ & $1(1)$ & $-8(1)$ & $-10(1)$ \\
$\mathrm{B}$ & $32(1)$ & $29(1)$ & $39(1)$ & $7(1)$ & $-14(1)$ & $-16(1)$ \\
$\mathrm{Cl}(1)$ & $79(1)$ & $25(1)$ & $91(1)$ & $2(1)$ & $-53(1)$ & $-6(1)$ \\
$\mathrm{O}(1)$ & $44(1)$ & $48(1)$ & $51(1)$ & $11(1)$ & $-20(1)$ & $-31(1)$ \\
$\mathrm{C}(1)$ & $31(1)$ & $29(1)$ & $30(1)$ & $5(1)$ & $-12(1)$ & $-12(1)$ \\
$\mathrm{O}(2)$ & $53(1)$ & $43(1)$ & $35(1)$ & $-5(1)$ & $-12(1)$ & $-18(1)$ \\
$\mathrm{C}(2)$ & $27(1)$ & $26(1)$ & $37(1)$ & $3(1)$ & $-12(1)$ & $-10(1)$ \\
$\mathrm{Cl}(2)$ & $60(1)$ & $49(1)$ & $60(1)$ & $16(1)$ & $-42(1)$ & $-23(1)$ \\
$\mathrm{C}(11)$ & $30(1)$ & $57(2)$ & $56(2)$ & $32(2)$ & $-1(1)$ & $-12(1)$ \\
$\mathrm{C}(12)$ & $36(1)$ & $80(2)$ & $30(1)$ & $-11(1)$ & $-1(1)$ & $-28(2)$ \\
$\mathrm{C}(13)$ & $30(1)$ & $30(1)$ & $57(2)$ & $-7(1)$ & $7(1)$ & $-10(1)$ \\
$\mathrm{C}(14)$ & $21(1)$ & $55(2)$ & $43(1)$ & $8(1)$ & $-9(1)$ & $-11(1)$ \\
$\mathrm{C}(15)$ & $30(1)$ & $43(2)$ & $61(2)$ & $-10(1)$ & $-1(1)$ & $-22(1)$ \\
\hline
\end{tabular}

Table 5. Hydrogen coordinates $\left(\times 10^{4}\right)$ and isotropic displacement parameters $\left(\AA^{2} \times 10^{3}\right)$ for $\mathbf{1}$.

\begin{tabular}{lcccc}
\hline & $x$ & $y$ & $z$ & $U_{\text {eq }}$ \\
\hline $\mathrm{H}(11 \mathrm{~A})$ & 3446 & 1632 & 4594 & 66 \\
$\mathrm{H}(12 \mathrm{~A})$ & 3285 & 4612 & 5193 & 62
\end{tabular}




\begin{tabular}{lllll}
$\mathrm{H}(13 \mathrm{~A})$ & 1844 & 6546 & 3304 & 57 \\
$\mathrm{H}(14 \mathrm{~A})$ & 1148 & 4834 & 1542 & 52 \\
$\mathrm{H}(15 \mathrm{~A})$ & 2181 & 1757 & 2257 & 57 \\
\hline
\end{tabular}

Table 6. Crystal data and structure refinement for $\left[\left({ }^{5}-\mathrm{C}_{5} \mathrm{H}_{5}\right) \mathrm{Fe}(\mathrm{CO})_{2} \mathrm{BCl}_{2} \cdot \mathrm{NC}_{5} \mathrm{H}_{4}-4-\mathrm{Me}\right](2)$.

Identification code

Empirical formula

Formula weight

Temperature

Wavelength

Crystal system

Space group

Unit cell dimensions

Volume

Z

Density (calculated)

Absorption coefficient

$\mathrm{F}(000)$

Crystal size

Theta range for data collection

Index ranges

Reflections collected

Independent reflections

Completeness to theta $=28.22^{\circ}$

Absorption correction

Max. and min. transmission

Refinement method

Data / restraints / parameters

Goodness-of-fit on $\mathrm{F}^{2}$

Final $\mathrm{R}$ indices [I $>2 \operatorname{sigma}(\mathrm{I})]$

$\mathrm{R}$ indices (all data)

Largest diff. peak and hole sad63m

C13 H12 B Cl2 Fe N O2

351.80

100(2) K

$71.073 \mathrm{pm}$

Monoclinic

$\mathrm{P} 2{ }_{1} / \mathrm{c}$

$\mathrm{a}=8.7555(11) \AA$

$\alpha=90^{\circ}$

$\mathrm{b}=11.6460(15) \AA$

$\beta=98.655(2)^{\circ}$

$\mathrm{c}=14.3163(18) \AA$

$1443.2(3) \AA^{3}$

4

$1.619 \mathrm{Mg} / \mathrm{m}^{3}$

$1.412 \mathrm{~mm}^{-1}$

712

$0.33 \times 0.32 \times 0.26 \mathrm{~mm}^{3}$

2.26 to $28.22^{\circ}$.

$-11<=\mathrm{h}<=11,-15<=\mathrm{k}<=15,-19<=\mathrm{l}<=18$

32192

$3473\left[\mathrm{R}_{\mathrm{int}}=0.0200\right]$

$97.6 \%$

Empirical

1.000 and 0.888

Full-matrix least-squares on $\mathrm{F}^{2}$

3473 / 0 / 229

1.065

$\mathrm{R} 1=0.0222, \mathrm{wR} 2=0.0565$

$\mathrm{R} 1=0.0228, \mathrm{wR} 2=0.0568$

0.380 and -0.292 e. $\AA^{-3}$

Table 7. Atomic coordinates [x $\left.10^{4}\right]$ and equivalent isotropic displacement parameters $\left[\AA^{2} \times 10^{3}\right]$ for $2 . U_{\text {eq }}$ is defined as one third of the trace of the orthogonalized $\mathrm{U}_{\mathrm{ij}}$ tensor.

\begin{tabular}{lcrrr}
\hline & $\mathrm{x}$ & $\mathrm{y}$ & $\mathrm{z}$ & \multicolumn{1}{c}{$\mathrm{U}_{\text {eq }}$} \\
\hline $\mathrm{Fe}$ & $7754(1)$ & $1080(1)$ & $1575(1)$ & $12(1)$ \\
$\mathrm{B}$ & $7587(2)$ & $4(1)$ & $2761(1)$ & $14(1)$ \\
$\mathrm{Cl}(1)$ & $9446(1)$ & $-13(1)$ & $3618(1)$ & $19(1)$ \\
$\mathrm{O}(1)$ & $7571(1)$ & $3049(1)$ & $2789(1)$ & $23(1)$ \\
$\mathrm{C}(1)$ & $7634(1)$ & $2258(1)$ & $2310(1)$ & $16(1)$ \\
$\mathrm{Cl}(2)$ & $7126(1)$ & $-1524(1)$ & $2407(1)$ & $19(1)$
\end{tabular}




\begin{tabular}{lrrrr}
$\mathrm{O}(2)$ & $4459(1)$ & $771(1)$ & $1026(1)$ & $29(1)$ \\
$\mathrm{C}(2)$ & $5767(2)$ & $893(1)$ & $1256(1)$ & $18(1)$ \\
$\mathrm{C}(11)$ & $10130(2)$ & $1161(1)$ & $1495(1)$ & $20(1)$ \\
$\mathrm{C}(12)$ & $9618(2)$ & $12(1)$ & $1366(1)$ & $18(1)$ \\
$\mathrm{C}(13)$ & $8461(2)$ & $-25(1)$ & $564(1)$ & $18(1)$ \\
$\mathrm{C}(14)$ & $8283(2)$ & $1108(1)$ & $176(1)$ & $19(1)$ \\
$\mathrm{C}(15)$ & $9307(2)$ & $1837(1)$ & $749(1)$ & $21(1)$ \\
$\mathrm{N}(21)$ & $6273(1)$ & $399(1)$ & $3368(1)$ & $14(1)$ \\
$\mathrm{C}(22)$ & $6576(2)$ & $1157(1)$ & $4086(1)$ & $17(1)$ \\
$\mathrm{C}(23)$ & $5437(2)$ & $1584(1)$ & $4558(1)$ & $18(1)$ \\
$\mathrm{C}(24)$ & $3908(2)$ & $1249(1)$ & $4301(1)$ & $16(1)$ \\
$\mathrm{C}(25)$ & $3611(2)$ & $450(1)$ & $3570(1)$ & $17(1)$ \\
$\mathrm{C}(26)$ & $4792(2)$ & $45(1)$ & $3125(1)$ & $16(1)$ \\
$\mathrm{C}(27)$ & $2636(2)$ & $1726(1)$ & $4778(1)$ & $21(1)$ \\
\hline
\end{tabular}

Table 8. Bond lengths [ $[\AA]$ and angles $\left[{ }^{\circ}\right]$ for $\mathbf{2}$.

\begin{tabular}{lc}
\hline $\mathrm{Fe}-\mathrm{C}(1)$ & $1.7421(13)$ \\
$\mathrm{Fe}-\mathrm{C}(2)$ & $1.7442(13)$ \\
$\mathrm{Fe}-\mathrm{C}(13)$ & $2.0981(13)$ \\
$\mathrm{Fe}-\mathrm{C}(11)$ & $2.1027(13)$ \\
$\mathrm{Fe}-\mathrm{C}(12)$ & $2.1082(13)$ \\
$\mathrm{Fe}-\mathrm{C}(14)$ & $2.1223(13)$ \\
$\mathrm{Fe}-\mathrm{C}(15)$ & $2.1230(13)$ \\
$\mathrm{Fe}-\mathrm{B}$ & $2.1326(14)$ \\
$\mathrm{B}-\mathrm{N}(21)$ & $1.6103(17)$ \\
$\mathrm{B}-\mathrm{Cl}(2)$ & $1.8774(14)$ \\
$\mathrm{B}-\mathrm{Cl}(1)$ & $1.8853(14)$ \\
$\mathrm{O}(1)-\mathrm{C}(1)$ & $1.1545(16)$ \\
$\mathrm{O}(2)-\mathrm{C}(2)$ & $1.1520(17)$ \\
$\mathrm{C}(11)-\mathrm{C}(12)$ & $1.4142(19)$ \\
$\mathrm{C}(11)-\mathrm{C}(15)$ & $1.431(2)$ \\
$\mathrm{C}(12)-\mathrm{C}(13)$ & $1.4133(19)$ \\
$\mathrm{C}(13)-\mathrm{C}(14)$ & $1.4307(18)$ \\
$\mathrm{C}(14)-\mathrm{C}(15)$ & $1.406(2)$ \\
$\mathrm{N}(21)-\mathrm{C}(22)$ & $1.3513(16)$ \\
$\mathrm{N}(21)-\mathrm{C}(26)$ & $1.3552(16)$ \\
$\mathrm{C}(22)-\mathrm{C}(23)$ & $1.3784(18)$ \\
$\mathrm{C}(23)-\mathrm{C}(24)$ & $1.3894(18)$ \\
$\mathrm{C}(24)-\mathrm{C}(25)$ & $1.3949(18)$ \\
$\mathrm{C}(24)-\mathrm{C}(27)$ & $1.4986(17)$ \\
$\mathrm{C}(25)-\mathrm{C}(26)$ & $1.3770(18)$ \\
$\mathrm{C}(1)-\mathrm{Fe}-\mathrm{C}(2)$ & \\
$\mathrm{C}(1)-\mathrm{Fe}-\mathrm{C}(13)$ & $96.19(6)$ \\
$\mathrm{C}(2)-\mathrm{Fe}-\mathrm{C}(13)$ & $97.41(6)$ \\
$\mathrm{C}(1)-\mathrm{Fe}-\mathrm{C}(11)$ & $98.50(6)$ \\
$\mathrm{C}(2)-\mathrm{Fe}-\mathrm{C}(11)$ & $66.00(5)$ \\
$\mathrm{C}(13)-\mathrm{Fe}-\mathrm{C}(11)$ & \\
& \\
& \\
&
\end{tabular}




\begin{tabular}{|c|c|}
\hline $\mathrm{C}(1)-\mathrm{Fe}-\mathrm{C}(12)$ & $131.95(6)$ \\
\hline $\mathrm{C}(2)-\mathrm{Fe}-\mathrm{C}(12)$ & $130.69(6)$ \\
\hline $\mathrm{C}(13)-\mathrm{Fe}-\mathrm{C}(12)$ & $39.27(5)$ \\
\hline $\mathrm{C}(11)-\mathrm{Fe}-\mathrm{C}(12)$ & $39.25(5)$ \\
\hline $\mathrm{C}(1)-\mathrm{Fe}-\mathrm{C}(14)$ & $126.86(5)$ \\
\hline $\mathrm{C}(2)-\mathrm{Fe}-\mathrm{C}(14)$ & $96.07(6)$ \\
\hline $\mathrm{C}(13)-\mathrm{Fe}-\mathrm{C}(14)$ & $39.63(5)$ \\
\hline $\mathrm{C}(11)-\mathrm{Fe}-\mathrm{C}(14)$ & $65.81(5)$ \\
\hline $\mathrm{C}(12)-\mathrm{Fe}-\mathrm{C}(14)$ & $65.93(5)$ \\
\hline $\mathrm{C}(1)-\mathrm{Fe}-\mathrm{C}(15)$ & $96.41(6)$ \\
\hline $\mathrm{C}(2)-\mathrm{Fe}-\mathrm{C}(15)$ & $127.11(6)$ \\
\hline $\mathrm{C}(13)-\mathrm{Fe}-\mathrm{C}(15)$ & $65.83(5)$ \\
\hline $\mathrm{C}(11)-\mathrm{Fe}-\mathrm{C}(15)$ & $39.60(5)$ \\
\hline $\mathrm{C}(12)-\mathrm{Fe}-\mathrm{C}(15)$ & $65.92(5)$ \\
\hline $\mathrm{C}(14)-\mathrm{Fe}-\mathrm{C}(15)$ & $38.68(5)$ \\
\hline $\mathrm{C}(1)-\mathrm{Fe}-\mathrm{B}$ & $87.92(5)$ \\
\hline $\mathrm{C}(2)-\mathrm{Fe}-\mathrm{B}$ & $87.12(6)$ \\
\hline $\mathrm{C}(13)-\mathrm{Fe}-\mathrm{B}$ & $104.57(5)$ \\
\hline $\mathrm{C}(11)-\mathrm{Fe}-\mathrm{B}$ & $104.84(5)$ \\
\hline $\mathrm{C}(12)-\mathrm{Fe}-\mathrm{B}$ & $85.02(5)$ \\
\hline $\mathrm{C}(14)-\mathrm{Fe}-\mathrm{B}$ & $144.19(5)$ \\
\hline $\mathrm{C}(15)-\mathrm{Fe}-\mathrm{B}$ & $144.44(5)$ \\
\hline $\mathrm{N}(21)-\mathrm{B}-\mathrm{Cl}(2)$ & $105.87(8)$ \\
\hline $\mathrm{N}(21)-\mathrm{B}-\mathrm{Cl}(1)$ & $105.57(8)$ \\
\hline $\mathrm{Cl}(2)-\mathrm{B}-\mathrm{Cl}(1)$ & $107.18(7)$ \\
\hline $\mathrm{N}(21)-\mathrm{B}-\mathrm{Fe}$ & $113.73(8)$ \\
\hline $\mathrm{Cl}(2)-\mathrm{B}-\mathrm{Fe}$ & $112.33(7)$ \\
\hline $\mathrm{Cl}(1)-\mathrm{B}-\mathrm{Fe}$ & $111.65(7)$ \\
\hline $\mathrm{O}(1)-\mathrm{C}(1)-\mathrm{Fe}$ & $178.79(11)$ \\
\hline $\mathrm{O}(2)-\mathrm{C}(2)-\mathrm{Fe}$ & $178.53(12)$ \\
\hline $\mathrm{C}(12)-\mathrm{C}(11)-\mathrm{C}(15)$ & $107.98(12)$ \\
\hline $\mathrm{C}(12)-\mathrm{C}(11)-\mathrm{Fe}$ & $70.59(7)$ \\
\hline $\mathrm{C}(15)-\mathrm{C}(11)-\mathrm{Fe}$ & $70.97(8)$ \\
\hline $\mathrm{C}(13)-\mathrm{C}(12)-\mathrm{C}(11)$ & $108.02(12)$ \\
\hline $\mathrm{C}(13)-\mathrm{C}(12)-\mathrm{Fe}$ & $69.98(7)$ \\
\hline $\mathrm{C}(11)-\mathrm{C}(12)-\mathrm{Fe}$ & $70.17(7)$ \\
\hline $\mathrm{C}(12)-\mathrm{C}(13)-\mathrm{C}(14)$ & $108.08(12)$ \\
\hline $\mathrm{C}(12)-\mathrm{C}(13)-\mathrm{Fe}$ & $70.75(7)$ \\
\hline $\mathrm{C}(14)-\mathrm{C}(13)-\mathrm{Fe}$ & $71.10(7)$ \\
\hline $\mathrm{C}(15)-\mathrm{C}(14)-\mathrm{C}(13)$ & $107.92(12)$ \\
\hline $\mathrm{C}(15)-\mathrm{C}(14)-\mathrm{Fe}$ & $70.69(8)$ \\
\hline $\mathrm{C}(13)-\mathrm{C}(14)-\mathrm{Fe}$ & $69.27(7)$ \\
\hline$C(14)-C(15)-C(11)$ & $107.98(12)$ \\
\hline $\mathrm{C}(14)-\mathrm{C}(15)-\mathrm{Fe}$ & $70.63(8)$ \\
\hline $\mathrm{C}(11)-\mathrm{C}(15)-\mathrm{Fe}$ & $69.43(7)$ \\
\hline $\mathrm{C}(22)-\mathrm{N}(21)-\mathrm{C}(26)$ & $117.73(11)$ \\
\hline $\mathrm{C}(22)-\mathrm{N}(21)-\mathrm{B}$ & $121.50(10)$ \\
\hline $\mathrm{C}(26)-\mathrm{N}(21)-\mathrm{B}$ & $120.58(10)$ \\
\hline $\mathrm{N}(21)-\mathrm{C}(22)-\mathrm{C}(23)$ & $122.28(12)$ \\
\hline $\mathrm{C}(22)-\mathrm{C}(23)-\mathrm{C}(24)$ & $120.59(12)$ \\
\hline
\end{tabular}




\begin{tabular}{ll}
$\mathrm{C}(23)-\mathrm{C}(24)-\mathrm{C}(25)$ & $116.67(11)$ \\
$\mathrm{C}(23)-\mathrm{C}(24)-\mathrm{C}(27)$ & $121.89(12)$ \\
$\mathrm{C}(25)-\mathrm{C}(24)-\mathrm{C}(27)$ & $121.45(12)$ \\
$\mathrm{C}(26)-\mathrm{C}(25)-\mathrm{C}(24)$ & $120.51(12)$ \\
$\mathrm{N}(21)-\mathrm{C}(26)-\mathrm{C}(25)$ & $122.19(12)$ \\
\hline
\end{tabular}

Table 9. Anisotropic displacement parameters $\left[\AA^{2} \times 10^{3}\right]$ for 2. The anisotropic displacement factor exponent takes the form: $-2 \pi^{2}\left[h^{2} a^{* 2} U_{11}+\ldots+2 h k a * b * U_{12}\right]$

\begin{tabular}{lrrrrrr}
\hline & $\mathrm{U}_{11}$ & $\mathrm{U}_{22}$ & $\mathrm{U}_{33}$ & $\mathrm{U}_{23}$ & $\mathrm{U}_{13}$ & $\mathrm{U}_{12}$ \\
\hline $\mathrm{Fe}$ & $12(1)$ & $13(1)$ & $12(1)$ & $0(1)$ & $2(1)$ & $1(1)$ \\
$\mathrm{B}$ & $13(1)$ & $15(1)$ & $15(1)$ & $0(1)$ & $2(1)$ & $0(1)$ \\
$\mathrm{Cl}(1)$ & $13(1)$ & $28(1)$ & $16(1)$ & $4(1)$ & $1(1)$ & $1(1)$ \\
$\mathrm{O}(1)$ & $28(1)$ & $18(1)$ & $24(1)$ & $-4(1)$ & $5(1)$ & $1(1)$ \\
$\mathrm{C}(1)$ & $15(1)$ & $17(1)$ & $16(1)$ & $3(1)$ & $3(1)$ & $1(1)$ \\
$\mathrm{Cl}(2)$ & $22(1)$ & $13(1)$ & $24(1)$ & $-1(1)$ & $7(1)$ & $0(1)$ \\
$\mathrm{O}(2)$ & $17(1)$ & $43(1)$ & $26(1)$ & $5(1)$ & $-2(1)$ & $-2(1)$ \\
$\mathrm{C}(2)$ & $19(1)$ & $20(1)$ & $14(1)$ & $2(1)$ & $2(1)$ & $1(1)$ \\
$\mathrm{C}(11)$ & $14(1)$ & $27(1)$ & $21(1)$ & $-3(1)$ & $6(1)$ & $-1(1)$ \\
$\mathrm{C}(12)$ & $17(1)$ & $21(1)$ & $18(1)$ & $2(1)$ & $6(1)$ & $5(1)$ \\
$\mathrm{C}(13)$ & $19(1)$ & $19(1)$ & $16(1)$ & $-3(1)$ & $6(1)$ & $2(1)$ \\
$\mathrm{C}(14)$ & $23(1)$ & $22(1)$ & $14(1)$ & $2(1)$ & $6(1)$ & $4(1)$ \\
$\mathrm{C}(15)$ & $23(1)$ & $18(1)$ & $24(1)$ & $1(1)$ & $12(1)$ & $-1(1)$ \\
$\mathrm{N}(21)$ & $14(1)$ & $14(1)$ & $13(1)$ & $1(1)$ & $3(1)$ & $-1(1)$ \\
$\mathrm{C}(22)$ & $17(1)$ & $18(1)$ & $17(1)$ & $-1(1)$ & $2(1)$ & $-4(1)$ \\
$\mathrm{C}(23)$ & $22(1)$ & $16(1)$ & $17(1)$ & $-3(1)$ & $5(1)$ & $-3(1)$ \\
$\mathrm{C}(24)$ & $20(1)$ & $14(1)$ & $17(1)$ & $4(1)$ & $7(1)$ & $1(1)$ \\
$\mathrm{C}(25)$ & $14(1)$ & $19(1)$ & $19(1)$ & $1(1)$ & $3(1)$ & $-2(1)$ \\
$\mathrm{C}(26)$ & $16(1)$ & $16(1)$ & $15(1)$ & $-1(1)$ & $2(1)$ & $-3(1)$ \\
$\mathrm{C}(27)$ & $21(1)$ & $20(1)$ & $24(1)$ & $-1(1)$ & $11(1)$ & $2(1)$ \\
\hline & & & & & &
\end{tabular}

Table 10. Hydrogen coordinates $\left(\times 10^{4}\right)$ and isotropic displacement parameters $\left(\AA^{2} \times 10^{3}\right)$ for $\mathbf{2}$.

\begin{tabular}{lrrrr}
\hline & $x$ & $y$ & $z$ & $U_{\text {eq }}$ \\
\hline$H(11 A)$ & 10880 & 1439 & 1967 & 29 \\
$\mathrm{H}(12 \mathrm{~A})$ & 9956 & -601 & 1766 & 19 \\
$\mathrm{H}(13 \mathrm{~A})$ & 7910 & -653 & 309 & 21 \\
$\mathrm{H}(14 \mathrm{~A})$ & 7610 & 1300 & -356 & 21 \\
$\mathrm{H}(15 \mathrm{~A})$ & 9410 & 2610 & 667 & 33 \\
$\mathrm{H}(22 \mathrm{~A})$ & 7610 & 1376 & 4215 & 21 \\
$\mathrm{H}(23 \mathrm{~A})$ & 5688 & 2081 & 5056 & 19 \\
$\mathrm{H}(25 \mathrm{~A})$ & 2630 & 160 & 3384 & 21 \\
$\mathrm{H}(26 \mathrm{~A})$ & 4611 & -483 & 2636 & 13 \\
$\mathrm{H}(27 \mathrm{~A})$ & 2020 & 1140 & 4960 & 34 \\
$\mathrm{H}(27 \mathrm{~B})$ & 1970 & 2190 & 4333 & 47 \\
$\mathrm{H}(27 \mathrm{C})$ & 3000 & 2135 & 5317 & 34 \\
\hline
\end{tabular}


